\title{
Design and Development of Agricultural Products Traceability System Based on Internet of Things RFID Technology
}

\author{
Hongsheng $\mathrm{Xu}^{1,}$ a and Yongliang $\mathrm{Li}^{1}$ \\ ${ }^{1}$ Luoyang Normal University, Luoyang, 471934, China \\ axhs_ls@sina.com
}

Keywords: RFID; Agricultural product; Traceability system; Internet of things; Tag

\begin{abstract}
This paper firstly analyzes the composition and working principle of the RFID system in Internet of things, and introduces the RFID tag, reader, middleware in detail. The traceability system of agricultural products is a system that tracks the agricultural products (including food, feed, etc.) into various stages of the market (from production to circulation). The paper presents design and development of agricultural products traceability system based on Internet of things RFID Technology. Experiments show that: RFID technology to achieve quality and safety management of agricultural products, fast and accurate tracking.
\end{abstract}

\section{Introduction}

Through the RFID technology in specific package of RFID tags containing with a valid identifier ID, and the ID is the world's only, through effective ID specific wireless reading device identification tags in the ROM to achieve on the label attached to object recognition [1]. RFID technology with characteristics of a globally unique ID makes this technology in the global scope of application of various industries in the range of continuous expansion. At present, RFID technology has become a powerful and efficient technical means to realize management information and improve the management level, enhance the core competitiveness of enterprises, and reduce the cost of enterprise operation.

The core of the Internet of things technology is RFID technology, so with the Internet of things technology, to solve the problem of food safety in society, it is particularly important. At present, the food safety traceability system based on RFID has been widely used in our country.

RFID technology in the implementation of security applications can be from many aspects the characteristics of to run blocking of fake goods in recent years this characteristic receives more and more attention of many experts, scholars and business circles; due to the continuous improvement of the RFID tag integration, manufacturing technology and label computing ability and storage capacity continues to increase, which makes RFID tag data security in addition to the password protection for data storage can also use with the complexity of encryption algorithm for data security management.

Agricultural product wholesale information is developed based on the development of a set of production records, information can be queried agricultural products traceability system [2]. This system can make business convenience will be its own high-quality agricultural products planting, picking, packing, the circulation link information display to the vast number of consumers, consumers can through smart phones to scan two-dimensional code can view the product the whole process from breeding to enter the market. The paper presents design and development of agricultural products traceability system based on Internet of things RFID Technology.

\section{Application of RFID Technology in Internet of Things}

Radio frequency identification RFID technology is a non-contact automatic identification technology based on radio frequency communication. It uses the radio frequency signal and its spatial coupling transmission characteristics to realize the automatic recognition of stationary or moving objects. Radio frequency identification is often referred to as an inductive electronic chip or a contactless card. 
With the development of RFID technology and the wide application of RFID network system based on networking sensor network and intelligent processing platform was increased in the existing traditional network infrastructure, the traditional network security measures have failed to provide reliable security, which appeared in the new security hidden danger [3]. RFID systems, there are privacy and authentication security risks, as is shown by equation(1), where $\mathrm{x}$ is in terms of privacy is mainly prevent attackers from the RFID tag of any form of illegal tracing; in the certification is to ensure that the label layer only with legitimate read write device communication.

$$
e_{i}^{*}=\frac{e_{i}}{\sigma}=\frac{y_{i}-\hat{y}_{i}}{\sigma}, i=1,2, \cdots, n
$$

Internet of things has its own distinctive characteristics. First of all, it is widely used in a variety of sensing technologies. Internet of things deployed a large variety of types of sensors, each sensor is a source of information, different types of sensors captured by the information content and information format is different. The data obtained by the sensor is real-time, according to a certain frequency of periodic acquisition of environmental information, and constantly update the data. Secondly, it is a ubiquitous network built on the Internet.

Low frequency system generally refers to its working frequency is less than $30 \mathrm{MHz}$, the typical operating frequency is: $125 \mathrm{KHz}, 225 \mathrm{KHz}, 13.56 \mathrm{M}$, etc., based on these frequency points of the radio frequency identification system generally have the corresponding international standard. The basic characteristic is that the cost of the electronic tag is low, the amount of data stored in the tag is less, and the reading distance is shorter, as shown in equation (2) [4].

$$
y_{i}=\left(1.8 e^{\frac{(t-10)}{1.5}}-1\right) \hat{y}_{i}, \mathrm{t} \geq 5
$$

RFID reader (Reader) is a device that interacts with the label information to read or write data and information on the label. The external antenna can be used for transmitting and receiving radio frequency signals, and can be divided into two types of portable or fixed type. The reader can be used as a single unit or as an embedded component in other systems, as shown in Fig. 1.

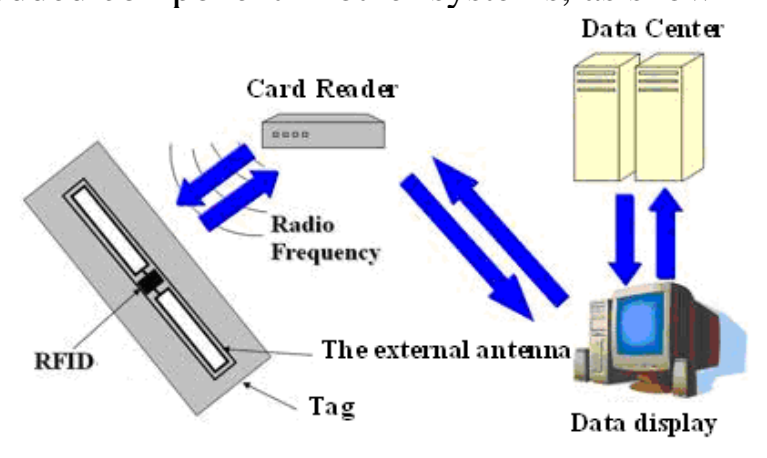

Figure 1. The working principle of RFID graph

Due to the limitations of RFID tag size and cost, the design of RFID system security and privacy solutions is a challenging research work, but also related to the RFID technology can further develop the application of the key [5]. Many articles discussed the security and privacy of RFID system, and proposed many schemes. According to the security mechanism, these schemes are divided into the physical mechanism scheme and the encryption scheme.

The reader generally includes a built-in antenna or an external antenna to increase the transmit power, and the general RFID reader should include at least one antenna (internal or external) to transmit or receive radio frequency signals, as is shown by equation(3), A read / write device is a task that is completed by a single antenna at the same time as a transmitter and a receiver, and a number of reading and writing devices are respectively carried out by the two antennas for transmitting and 
receiving [6]. The number and form of antenna used in the reader should be combined with the specific application.

$$
x^{(0)}=\left(x^{(0)}(1), x^{(0)}(2), x^{(0)}(3), x^{(0)}(4), x^{(0)}(5), x^{(0)}(6), x^{(0)}(7), x^{(0)}(8), x^{(0)}(9), x^{(0)}(10)\right)
$$

Electronic label is stored in the RFID system is identified object related information of the electronic device, usually attached to the surface or embedded within the to be identified, tag memory information may have read write device of non-contact reading and writing. The electronic tag is composed of 4 parts, an antenna, a control module, a memory and a receiving and sending module.

The information carrier of the traditional bar code identification technology is the paper, so it is easy to be polluted and easily damaged. As the RFID tag will chip as a data carrier, so the water, oil, chemicals and other substances on the corrosion of its impact on pollution is small, suitable for a variety of applications, and read a good performance.

\section{Design of Agricultural Product Traceability System}

Anti - counterfeiting technology: refers to the goods to achieve the purpose of anti - counterfeiting, in a certain range can accurately identify the authenticity is not easy to be copied and copied technology. Generally speaking, anti-counterfeiting technology should has some features: difficult to copy and imitation, the product anti-counterfeiting technology complex degree, high anti fake product itself, the price is reasonable, to verify the authenticity of a product easy to test, have within a certain time limit cannot be third party successfully copied or replication costs are too high.

Agricultural products traceability system by recording and display of agricultural products of the basic information of the products, product introduction, planting the recording process, picking, packing, transportation process, origin, quality inspection, certificate of honor and the details to achieve to enhance the value of the products and consumer trust of the brand, the system covers the vegetables, fruits, aquatic products, grain, meat, non-staple food, dry goods, tea, edible fungus nine categories of agricultural products, covering a wide range of simple operation, as is shown by equation(4) [7].

$$
v_{k}(t)=e^{-\lambda t} \frac{(\lambda t)^{k}}{k !}, k=1,2,3, \cdots
$$

Because of the complexity of the various agricultural products planting (breeding), production (processing), storage, transportation, sales and other links, and is not standardized, both of similar agricultural products (rice) of different enterprises, and its production process, product storage, transportation, packaging, and other links will have great difference [8]. Therefore, it is to the agricultural product implementation process monitoring, tracing, and tracking.

Traceability system is in the whole process of product supply all relevant information of the product were stored records quality guarantee system, its purpose is able to quickly and efficiently query to the problem of raw materials or steps in the process in the product quality problems, when necessary for product recalls, was carried out in the punitive measures, thus to improve the level of product quality. It is helpful to the quality control and recalls the products when necessary.

\section{Development of Agricultural Products Traceability System Based on Internet of Things RFID Technology}

RFID technology in the field of anti-counterfeiting caused concern at home and abroad widely, it is relative to the traditional security technology Traceability Technology with obvious advantages, it has a non contact, multi-objective, high efficiency, automatic recognition of characteristics [9]. The registration system of enterprise registration is simple, and the process of false proof authentication and traceability information input are completed automatically, so that the anti fake label data is not easy to be forged, and the environment adaptability is strong. RFID security if combined with other 
anti-counterfeiting technology, but also to enhance the effect of anti-counterfeiting. The traceability process from commodity production enterprise sectors, to achieve safe packaging, through reading and writing device between the label and the mutual authentication mechanism set up the circulation of commodities throughout the whole process of electronic information history, establishment of product traceability system.

Agricultural products traceability system design:

Step1: on the agricultural products traceability information with written RFID tag card;

Step2: through the RFID reader will be contained within the agricultural product quality safety traceability information read into the computer;

Step3: through a simple web service components designed to find the corresponding EPC code corresponding to the web detailed information. The readable and writable tags in addition to the tag ID (UID) never variable can read and write tag other data can be due to the need to update, and according to the label ID number can only determine the label, and only you can determine the corresponding agricultural products.

Design of RFID agricultural products traceability system, can be accessed through the internet. It is divided into three levels - application server layer, data management layer, and client layer. Application server layer provides client access to business logic and user interface. Data management is composed of the database itself and its database management system [10]. Client layer can be accessed through the hypertext transfer protocol (HTTP) through a simple object access protocol (SOAP), as is shown by equation (5).

$$
\lambda p_{2}=3 u p_{3}, p_{3}=\left(\frac{\lambda}{u}\right)^{3} \frac{p_{0}}{3 !}=\frac{\rho^{3}}{3 !} p_{0}
$$

Agricultural products traceability system integrated use of the variety of network technology and two-dimensional code recognition of cutting-edge technology, implementation of records on production of agricultural products throughout the electronic network management, create a transparent "status file" to appear on the market of agricultural products. Consumers in the purchase of the agricultural products, only need to use the phone in alignment product packaging the pasted "traceability of agricultural products specific identification codes" label on the two-dimensional code scanning, it will immediately know purchased the product name, nutrition composition, size, long shelf life, storage precautions, growth cycle, production enterprises, production base location and production process the application of pesticides, fertilizers etc. a full set of production profile.

RFID technology to build the agricultural products traceability system based on agricultural production and operation organizations provide membership registration, the traceability of the service application, product information dissemination services to provide business operation backstage and it gathers the information management, as is shown by Fig. 2 .

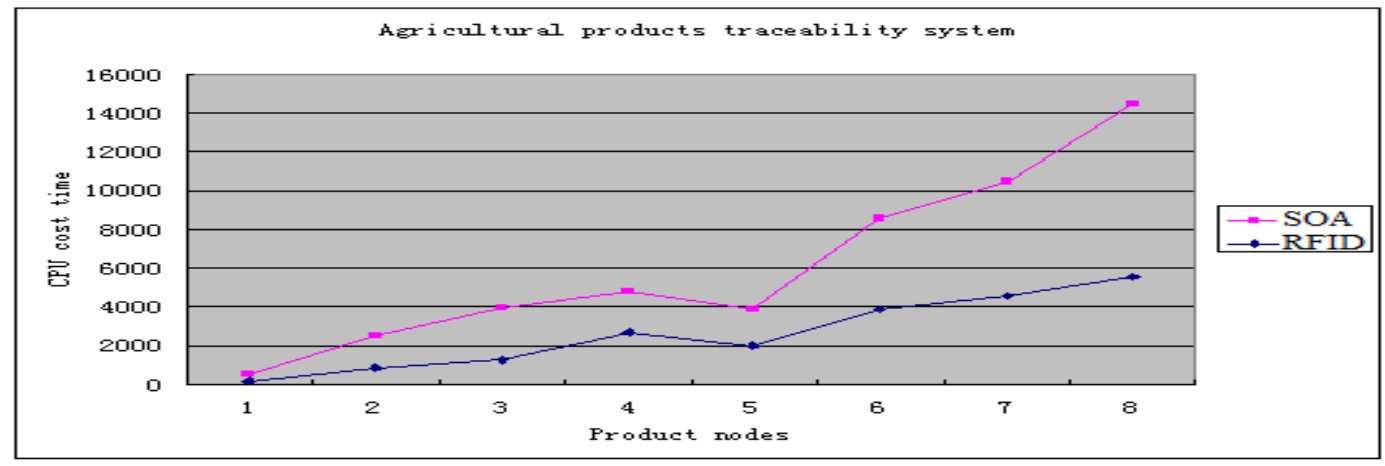

Figure 2. Development of agricultural products traceability system based on Internet of things RFID Technology 
Based on SOA to construct agricultural products traceability system is mainly through the study of corresponding components and applications to compose new services and for different agricultural products production and processing enterprises, flexible construction application and traceability procedure. By adopting the SOA framework, the agricultural production enterprises can reduce the coupling between systems, and improve the reusability of the system.

\section{Summary}

The paper presents design and development of agricultural products traceability system based on Internet of things RFID Technology. RFID technology as with multiple target recognition, non contact identification and no artificial intervention and other advantages, in this paper, the various modules of RFID traceability system design, eventually by the entry page. Simulation and realized the design of the main function modules of the prototype system.

\section{Acknowledgements}

This paper is supported by the science and technology research major project of Henan province Education Department (13B520155), Henan Province basic and frontier technology research project (142300410303) and Research project of teaching reform in Luoyang Normal University (2015-XJJG-041).

\section{References}

[1] Barnaghi P, Wang W, Henson C, et al, Semantics for the Internet of Things: early progress and back to the future, International Journal on Semantic Web and Information Systems, vol.8, no.1, (2012),pp.11-21.

[2] Thirumalai T., Kashwan K. R., New Reader to Reader Anti-Collision Protocol for Mobile and Dense RFID Reader Environment: A TDMA based Approach, IJACT, Vol. 7, No. 4, pp. 10 21, 2015.

[3] Haishui Jin, Guochen Wei, Jun Wu, Study on the Application of Information Coding and EAN.UCC Bar Code in Agricultural Product Quality Traceability — Based on the Analysis of Agricultural Supply Chain, JCIT, Vol. 8, No. 10, pp. $583 \sim$ 591, 2013.

[4] Pfisterer D, Romer K, Bimschas D, et al, SPITFIRE: toward a semantic Web of things, Communications Magazine, vol.49, no.11, (2011),pp.40-48.

[5] Feng Feng, Xu Qi, Locating Method in the Warehouse Based on Active RFID Tags, JDCTA, Vol. 7, No. 1, pp. $223 \sim 231,2013$.

[6] Md. Motaharul Islam, Pham Phuoc Hung, Al - Amin Hossain, Mohammad Aazam, , A Framework of Smart Internet of Things based Cloud Computing, RNIS, Volume 14, pp. 646 651, 2013.

[7] Hongsheng $\mathrm{Xu}$, Ruiling Zhang, Chunjie Lin, Youzhong Ma, Novel Approach of Semantic Annotation by Fuzzy Ontology based on Variable Precision Rough Set and Concept Lattice, International Journal of Hybrid Information Technology Vol.9, No.4 (2016), pp. 25-40.

[8] Xue-jun Zhang, Chuan-ling Ye, Jun-fei Ma, An Improved Anti-collision Algorithm with Intelligent Separation for RFID System, IJACT, Vol. 4, No. 22, pp. $823 \sim$ 831, 2012.

[9] Hongsheng Xu, Ruiling Zhang, Novel Approach of Fault Diagnosis in Wireless Sensor Networks Node Based On Rough Set and Neural Network Model, International Journal of Future Generation Communication and Networking Vol. 9, No. 4 (2016), pp. 1-16. 
[10] Yongfeng Wang, Yu Yang, Yongming Gu, Research on Quality and Safety Traceability System of Fruit and Vegetable Products Based on Ontology, JCIT, Vol. 7, No. 1, pp. 86 93, 2012. 